\title{
A Hybrid WLAN-Bluetooth Access Network Solution for a More Efficient VoIP-Data \& Video Traffic Management ${ }^{\star}$
}

\author{
David Pérez ${ }^{1}$, José Luis Valenzuela ${ }^{1}$, Ángela Hernández ${ }^{2}$, Antonio Valdovinos ${ }^{2}$ \\ 1 Universitat Politècnica de Catalunya (UPC), \\ Avda.Canal Olimpic s/n, (08860) Castelldefels, SPAIN \\ dperez@tsc.upc.edu, valens@tsc.upc.edu \\ 2 Institute of Engineering in Aragón, I3A, University of Zaragoza, \\ María de Luna 1, (50018) Zaragoza, SPAIN \\ anhersol@unizar.es, toni@unizar.es
}

\begin{abstract}
In this paper we present a hybrid Physical and Medium Access Control protocol for a Wireless LAN which is designed to support both synchronous (voice) and asynchronous (data) traffic. The protocol is designed using a modified Bluetooth core and $802.11 \mathrm{e}$ radio access, where the voice service is supported by Bluetooth whereas data and video services are provided by $802.11 \mathrm{e}$. The Bluetooth radio access is modified to operate using an orthogonal frequency hopping radio in bands of $20 \mathrm{MHz}$ coincident with 802.11 e channels.
\end{abstract}

Keywords. Bluetooth, WLAN, MAC.

\section{Introduction}

The evolution of communication systems in last years has followed a clear tendency towards B3G systems (Beyond Third Generation) or 4G, that are intended to provide a clear support to the integration and coexistence of multiple and different Radio Access Technologies (RATs) in a unique and complex radio environment assuming the management of the whole system and coordinating the radio access with the Core Network. This core, the IP Multimedia Subsystem (IMS), provides the control functionalities and an ubiquitous and seamless wireless access system 2G (GSM), 3G (UMTS) and wide band (WLAN, WiMax), extended to auto organized network schemes with short range connectivity (Bluetooth) between intelligent terminals.

Wireless local area networks have been predominantly used to support data applications. However recent developments, particularly the ability of personal computers ( $\mathrm{PCs}$ ) to deal with real-time voice, have demonstrated the need for

\footnotetext{
* This work has been supported by CYCIT (Spanish National Science Council) under the grant TEC2006-09109, which is partially financed from the European Community through the FEDER program.
}

Please use the following format when citing this chapter:

Pérez, D., Luis Valenzuela, J., Hernádez, Á., Valdovinos, A., 2007, in IFIP International Federation for Information Processing, Volume 245, Personal Wireless Communications, eds. Simak, B., Bestak, R., Kozowska, E., (Boston: Springer), pp. 133-144. 
wireless LANs to efficiently support both voice and data traffic. Lately voice communications are heading to use data networks; usually IP networks. This kind of communications is known as VoIP. The requirements of voice and data traffic are however very different. In general voice networks can tolerate errors and packet loss $(<1-5 \%)$ without degrading service, but have real-time constraints and fixed assignment wireless protocols are more appropriate such Time Division Multiple Access (TDMA). On the other hand data networks can tolerate packet delays, but cannot allow errors or packet losses, and random access protocols are more appropriate. Thus protocols designed for data networks typically use some form of CSMA/CA. Our goal is to be able to support high quality real-time voice conversations and, at the same time, provide a high data throughput. To overcome the limitations of current MAC technologies we have been investigating how to keep the advantages of both types of MAC protocols, without reducing the quality of service for each traffic type, and without increasing the overall complexity of the system. During system design a decision is taken to optimally support one or other traffic type and this leads to the adoption of a particular access mechanism.

In this work we propose a hybrid WLAN-Bluetooth Radio Access System, which allows taking profit of WLAN and Bluetooth technologies by defining an adaptive MAC implementation. The interaction in a multi system WLANBluetooth network can facilitate, for example, coexistence of voice and data services. The first technology (WLAN), based on CSMA/CA is appropriate for data communications and the second one, based on TDMA, for voice communications. The main idea is to share radio resources among WLAN and Bluetooth, in a superframe time base of $20 \mathrm{~ms}$, divided in two parts. In the first part, a BT-TDMA access is employed by users with VoIP traffic demand. In the second one, a WLAN CSMA/CA access is employed by users with data traffic demands. The number of resources, assigned to WLAN and BT access, is not fixed but it depends on VoIP users. On the other hand, to provide high quality voice we have chosen to use an AMR voice codec which is identical to the one used by UMTS. This codec is widely used and enables us to offer good integration with the telephony network. Adaptive Multi-Rate (AMR) codecs are standardized by 3GPP for GSM [1] [2] the world's most widespread cellular technology, as well as for WCDMA and a payload format for IP-transport has also been standardized in IETF [3]. The paper is organized as follows. First, fundamentals of $802.11 \mathrm{e} / \mathrm{g}$ and Bluetooth standard are briefly explained before introducing a detailed description of the proposal. In Section 3, the proposed hybrid Physical and Medium Access Control protocol is presented and simulation results obtained are discussed. Finally, conclusions are summarized in Section 5.

\section{Fundamentals of Bluetooth and 802.11e/g}

\section{$2.1 \quad 802.11$}

Since the appearance of the initial IEEE 802.11 standard [4], near ten years ago, wireless networks have experienced a vertiginous evolution. But, to be able 
to support voice and multimedia applications demanded by the market, just increasing transmission rates is not enough. It is also necessary a good management of these resources and to be able to offer to users what is called quality of service (QoS). Task group E of 802.11 IEEE working group, developed a standard [5] able to differentiate several traffic types based on the different QoS needs. This standard has evolved the original Distributed Coordination Function (DCF) and Point Coordination Function (PCF) to the new Enhanced Distributed Channel Access (EDCA) and Hybrid Coordination Function (HCF) Controlled Channel Access. The main difference is the establishment of four Access Categories (AC). Depending on the category to which a packet belongs, it has more or less priority. Fig. 1 shows the basic procedure.

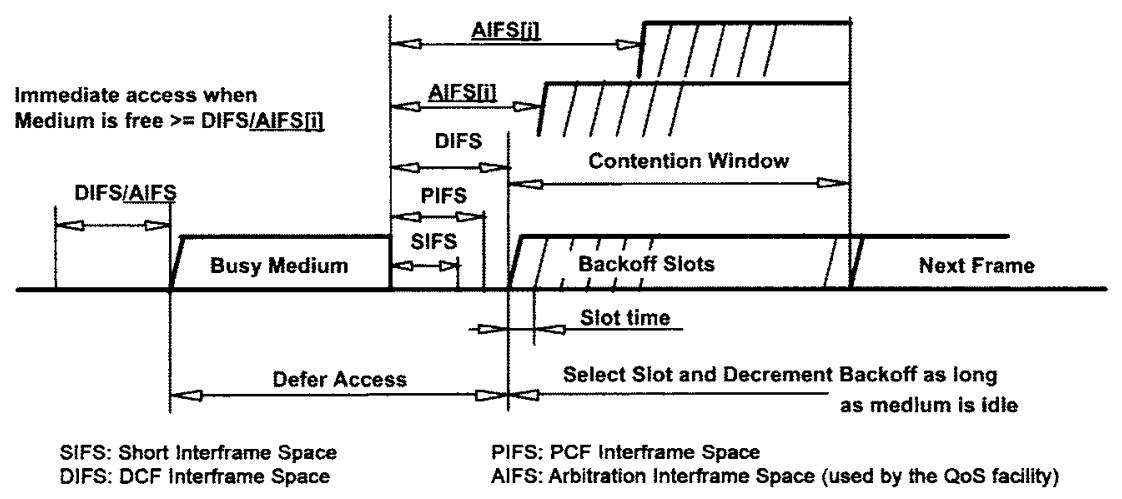

Fig. 1. 802.11e Back-off procedure

The 802.11g [6] standard proposes an extension of the transmission rates up to $54 \mathrm{Mbps}$. The physical layer of 802.11 is modified and uses an OFDM modulation. At MAC layer level operation is the same as 802.11 , so the medium access mechanism is CSMA/CA. The standard was designed to be backward compatible; so, the possibility of working in mixed mode is also considered $(802.11 \mathrm{~b}+802.11 \mathrm{~g})$. Nevertheless, we will use only the OFDM-PHY rates of $802.11 \mathrm{~g}$, that is, from 6 to $54 \mathrm{Mbps} .802 .11 \mathrm{e}$, however, modifies the legacy 802.11 MAC. It establishes four AC: Voice, Video, Best effort and Background.

In each one of these access categories a set of parameters is defined that depends on the traffic priority. These parameters are also different for the Access Point (see Table 1). And they are:

- AIFS[i]: it indicates the minimum number of delay slots before the contention window for each access category, as it is possible to observe in Fig.1.

- CWmin and CWmax: the size of the contention window will depend on the AC assigned.

- Transmission Opportunity (TXOP): once won the access to the channel, a station is allowed to send packets as long as the duration of them is smaller 
than the remaining TXOP. If TXOP is zero the station is only allowed to send one packet.

Table 1. Default $802.11 \mathrm{e}$ and $802.11 \mathrm{~g}$ parameters

\begin{tabular}{cccc} 
Parameter AV-Voice(STA) & AC-Voice(AP) & AC-Best effort \\
\hline \hline AIFSN & 2 & 1 & 3 \\
CWmin & 3 & 3 & 15 \\
CWmax & 7 & 7 & 1023 \\
TXOP & $1054 \mu s$ & $1054 \mu s$ & 0 \\
\hline \hline PHY & \multicolumn{4}{c}{ ERP-OFDM }
\end{tabular}

The effective time to transmit a data packet and supposing that there are no collisions can be computed following (1) and Fig.2.

$$
T_{e f}(\mu s)=T_{\text {Access }}+T_{P H Y}+T_{D A T A}+T_{A C K}
$$

where,

$$
\begin{aligned}
& T_{a c c e s s}=D I F S+\frac{C W_{\min }}{2} \cdot S I F S \\
& T_{P H Y}=P L C P_{\text {preamble }}+\text { Signal }+ \text { Signal } \text { Ext }_{\text {ig }} \\
& \text { Headers }=M A C_{h}+I P_{h}+U D P_{h}+R T P_{h} \\
& T_{D A T A}=\left\lceil\frac{\text { Service }+ \text { Tail }+8\left(\text { Headers }+M A C_{C R C}+D A T A\right)}{N_{D B P S}}\right\rceil \cdot T_{\text {sym }} \\
& T_{A C K}=T_{P H Y}+\left\lceil\frac{M A C_{A C K} \cdot 8+\text { Service }+ \text { Tail }}{N_{D B P S}}\right\rceil \cdot T_{\text {sym }}
\end{aligned}
$$

and $N_{D B P S}$ is the number of bits per OFDM symbol and depends on the transmission rate. All the values are shown on Table II. And the operator ceiling $(\lceil\rceil)$ returns the smallest integer greater than or equal the specified number.

Table 2. Considered parameters

\begin{tabular}{cc|cc|cc} 
Parameter & Value & Parameter & Value & PHY rate & $N_{D B P S}$ \\
\hline \hline MAC header & 24 bytes & Tail bits & 6 bits & $6 \mathrm{Mbps}$ & 24 \\
MAC CRC & 4 bytes & PLCP preamble & $16 \mu s$ & $9 \mathrm{Mbps}$ & 36 \\
MAC ACK & 14 bytes & Signal & $4 \mu s$ & $12 \mathrm{Mbps}$ & 48 \\
IP header & 20 bytes & Signal Extension & $6 \mu s$ & $18 \mathrm{Mbps}$ & 72 \\
UDP header & 8 bytes & Slot Time & $9 \mu s$ & $24 \mathrm{Mbps}$ & 96 \\
RTP header & 12 bytes & SIFS & $10 \mu s$ & $36 \mathrm{Mbps}$ & 144 \\
AMR Data & 31 bytes & AIFS & $28 \mu s$ & $48 \mathrm{Mbps}$ & 192 \\
Service bits & 16 bits & $T_{\text {sym }}$ & $4 \mu s$ & $54 \mathrm{Mbps}$ & 216
\end{tabular}




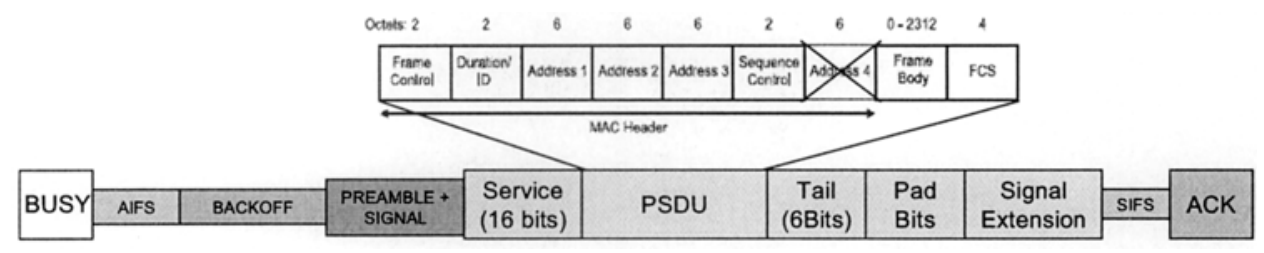

Fig. 2. 802.11 packet transmission structure

\subsection{Bluetooth}

Bluetooth [7] is a universal radio interface in the $2.4 \mathrm{GHz}$ ISM frequency band. Bluetooth is based on a centralized connection oriented approach. Bluetooth devices sharing a wireless channel form a piconet. One device in a piconet has the role of master and controls the channel access, while the others are slaves. There may be up to seven slaves in a piconet.

Bluetooth uses a Time-Division Duplex (TDD) scheme to divide the channel into $625 \mathrm{~ms}$ time slots. Master and slave units transmit alternately. Each piconet is characterized by a particular fast frequency-hopping pattern; the frequency is uniquely determined by the master's address and is followed by all the devices participating in the piconet.

Two types of connections can be established in a piconet: the Synchronous Connection-Oriented (SCO) link, and the Asynchronous Connectionless (ACL) link. SCO links provide a circuit-oriented service with constant bandwidth based on a fixed and periodic allocation of slots. They require a pair of slots once every two, four or six slots, depending upon the SCO packet used. ACL connections, on the other hand, provide a packet-oriented service and span over one, three or five slots. For ACL links, Bluetooth uses a fast acknowledgment and retransmission scheme to ensure reliable transfer of data. The master controls traffic on ACL links by employing a polling scheme to divide the piconet bandwidth among the slaves. A slave is only allowed to transmit after the master has polled it.

SCO links have been designed to support voice services. Since these links require a periodic allocation of a pair of slots once every two, four or six slots. The SCO link is a symmetric, point-to-point link between the master and a single slave in the piconet. The SCO link involves reservation of slots and can therefore be considered as a circuit-switched connection between master and slave. The master can support up to three SCO links to the same slave or to different slaves. SCO packets have been designed to support $64 \mathrm{kbps}$ speech. The specifications define three pure SCO packets and one hybrid SCO packet, which carries an asynchronous data field in addition to a synchronous voice field.

The ACL link provides a packet-switched connection between the master and all active slaves in the piconet. A slave can send an ACL packet if it has been addressed by the master in the previous slot. To ensure data integrity, ACL packets are retransmitted. Only a single ACL link can exist between a master 
and a slave. The master schedules ACL packets in the slots not reserved for the SCO links. The specifications define several kinds of ACL packets, see Table 3.

Table 3. ACL Packets

\begin{tabular}{|c|c|c|c|c|c|c|c|}
\hline $\begin{array}{c}\text { Packet } \\
\text { type }\end{array}$ & $\begin{array}{l}\text { Time } \\
\text { Slots }\end{array}$ & Modulation & $\begin{array}{c}\text { Payload } \\
\text { (user bytes) }\end{array}$ & $\begin{array}{c}\text { Packet } \\
\text { type }\end{array}$ & $\begin{array}{l}\text { Time } \\
\text { Slots }\end{array}$ & Modulation & $\begin{array}{c}\text { Payload } \\
\text { (user bytes) }\end{array}$ \\
\hline$\overline{\mathrm{DM} 1}$ & $\overline{1}$ & $\overline{\text { GFSK }}$ & $0-17$ & $2-\mathrm{DH} 1$ & 1 & $\pi / 4 D Q P S K$ & $0-54$ \\
\hline DH1 & 1 & GFSK & $0-27$ & 2-DH3 & 3 & $\pi / 4 D Q P S K$ & $0-367$ \\
\hline DM3 & 3 & GFSK & $0-121$ & 2-DH5 & 5 & $\pi / 4 D Q P S K$ & $0-679$ \\
\hline DH3 & 3 & GFSK & $0-183$ & 3-DH1 & 1 & $8 P S K$ & $0-83$ \\
\hline DM5 & 5 & GFSK & $0-224$ & 3-DH3 & 3 & $8 P S K$ & $0-552$ \\
\hline DH5 & 5 & GFSK & $0-339$ & $3-\mathrm{DH} 5$ & 5 & $8 P S K$ & $0-1021$ \\
\hline AUX & 1 & GFSK & $0-29$ & & & & \\
\hline
\end{tabular}

Bluetooth devices communicate with each other by using standard networking protocols to transport control and data packets over a network. Devices may use protocols such as TCP, IPv4 or IPv6. These protocols have dissimilar network packet formats. To provide seamless transmission of network packets over the L2CAP layer in the protocol stack, an intermediate protocol is required that encapsulates dissimilar network packet formats as a standard common format.

The Bluetooth Network Encapsulation Protocol (BNEP) provides this encapsulation by replacing the networking header, such as an Ethernet header, with a BNEP header. The L2CAP layer encapsulates the BNEP header and the network payload and sends it over the transport media. The Bluetooth Personal Area Networking profile describes how BNEP shall be used to provide networking capabilities for Bluetooth devices.

\section{The hybrid physical-MAC protocol proposal}

Once basic principles of WLAN and BT have been introduced, we describe the protocol of the physical-MAC level we propose. It is essentially a hybrid protocol (adaptive MAC) which combines both 802.11 and Bluetooth access mechanisms, Fig.3, in order to take profit of the advantages of both technologies.

Hybrid MAC protocols are not unique [8], [9] but the protocol described here incorporates features which ensure good performance under a wide range of conditions. The hybrid MAC protocol uses a superframe (whose duration is fixed to $20 \mathrm{~ms}$ ), which incorporates a contention free period (CFP) and a contention period. The access mechanism used during each CFP is TDMA (modified Bluetooth access), whereas the access mechanism used during the contention period is CSMA/CA. The first one will be used for VoIP communications (with codecs selected appropriately) and the second one, for data communications. The total amount of resources is distributed on an adaptive bandwidth partitioning strategy between Bluetooth and WLAN, depending on the number of VoIP users. The main idea is illustrated on Fig.4. However, before continuing describing 


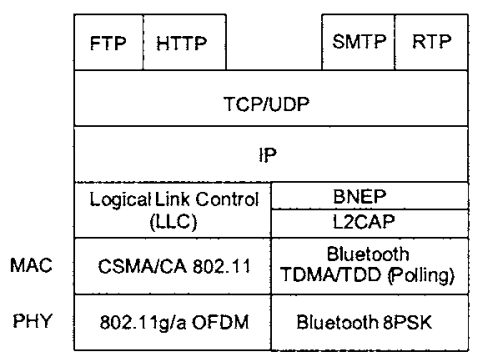

Fig. 3. Protocol stack

with more detail the MAC implementation and its associated parameter values, we review some aspects related to the required network architecture, the physical layer, the traffic sources and the associated framing structures.

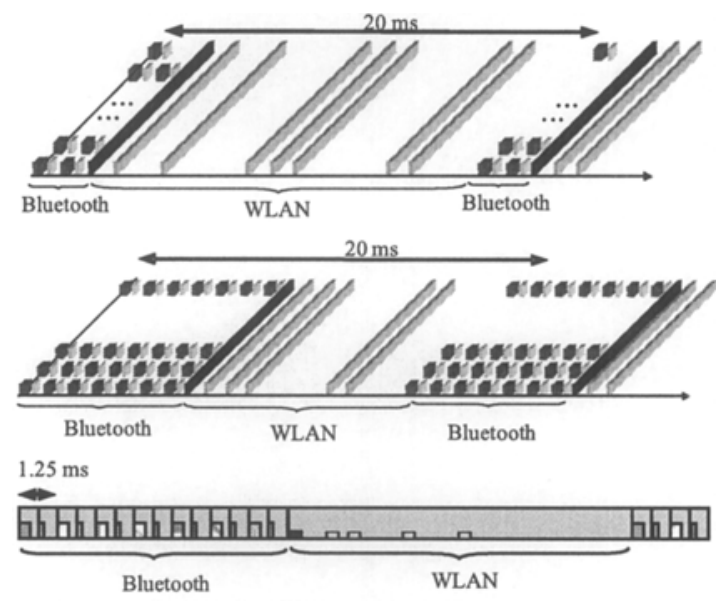

Fig. 4. Time-frequency proposed structure scheme

\subsection{Network architecture}

To support the proposed scheme, the network architecture consists of:

- Access Point (AP) that supports voice and data services.

- Voice Terminal that only uses the TDMA access mechanism to communicate with the Access Point.

- Data Node that uses the CSMA/CA access mechanism and can communicate with an Access Point and other data nodes.

- Voice and Data Node which can use both access mechanisms. 


\subsection{Physical layer}

At the physical level, the system is designed to operate in the $2.4 \mathrm{GHz}$ ISM band, but Dynamic Frequency Selection (DFS) can be used to allow the possibility of using the $5 \mathrm{GHz}$ band. When working in WLAN mode, the behavior of the radio hardware is the same as an off the shelf $802.11 \mathrm{~g}$ WLAN using the ERP-OFDM PHY layer that employs the whole $20 \mathrm{MHz}$ channel. On the other hand when working in Bluetooth mode, the specifications indicate that a Bluetooh receiver requires $5 \mathrm{~dB}$ of Adjacent $(1 \mathrm{MHz})$ interference $C / I_{1 M H z}$ for $8 \mathrm{PSK}$ modulation and $-25 \mathrm{~dB}$ Adjacent $(2 \mathrm{MHz})$ interference $C / I_{2 M H z}[7]$. However commercial Bluetooth modules achieve a $C / I_{2 M H z}$ up to $-40 \mathrm{~dB}$ [10]. So, with a band separation of $2 \mathrm{MHz}$ a right operation is guaranteed. Thus we propose that the radio hardware used works with ten new frequency hopping sequence sets in bands of $1 \mathrm{MHz}$ with a guard band of $1 \mathrm{MHz}$. This guard band was considered instead of a continuous band in order to reduce adjacent interference when several frequencies have been used simultaneously. And moreover, the resultant spectrum is similar to the one of a WLAN.

\subsection{Traffic and framing structure}

We consider the VoIP transfer over Bluetooth using Bluetooth Network Encapsulation Protocol (BNEP). For that reason we looked for one of the voice codecs with smaller size, AMR codec [1]. The encoder outputs compressed speech data in octet aligned (by using bit stuffing) AMR-NB Interface Format 2, as defined in the 3GPP TS 26.201 [11]. Other codecs as can be $i L B C$, used in Skype, have similar characteristics.

The use of BNEP for transporting a voice ethernet packet is shown in Fig. 5. $B N E P$ removes and replaces the ethernet header with the BNEP header. Finally, both the BNEP header and the payload are encapsulated by L2CAP and are sent over the Bluetooth physical channel.

\begin{tabular}{|c|c|c|c|}
\hline L2CAP Header & BNEP Header & IP/UDP/RTP & Payload \\
\hline 4 Bytes & 4 Bytes & 40 Bytes & $13-31$ Bytes
\end{tabular}

Fig. 5. Voice packet structure over L2CAP and BNEP

Bluetooth offers several types of packets with different user payloads and rates. We propose the use of $3 \mathrm{DH} 1$ packets to transport the encapsulated voice payload. For example, if we use the seventh mode of the AMR codec it has a bit rate of $12.2 \mathrm{kbps}$. Thus, we generate a 31 bytes packet each $20 \mathrm{~ms}$, moreover we have to add to this 20 bytes of the IP protocol header plus eight of the UDP header, four of the BNEP and four of L2CAP. Looking at Table 3 we can see that it is possible to use the $3 \mathrm{DH} 1$ mode or even the $2 \mathrm{DH} 1$ mode for lower rate codecs. 
With respect to data traffic, transmission over $802.11 \mathrm{~g}$ uses the packet structure shown before in Fig.2, follows the access mechanism of Fig.1 and its default parameters were shown in Table 2.

\subsection{The MAC Protocol}

The start of the superframe is the point at which voice stations start to transmit. The duration of the superframe is fixed and equal to $20 \mathrm{~ms}$. This dwell period is fixed at $20 \mathrm{~ms}$ to provide acceptable performance with respect to latency.

The length of the dwell period also means that each voice data message contains $20 \mathrm{~ms}$ of AMR data. In addition each packet transmitted includes the necessary MAC and PHY headers. Each one of the contention free periods is divided into a number of pairs of fixed length slots $(625 \mathrm{~ms})$, two per voice connection. The first slot in each pair is used to transmit voice data from the Access Point to a node (downlink) and the second is used to transmit voice data from a node to the Access Point (uplink) as shown in Fig.4.

Each Bluetooth station acting as Master can address up to seven clients simultaneously. As we have proposed the standard is extended to allow ten orthogonal hopping sequences. This implies we can handle up to ten voice users per each pair of slots that is a maximum of 70 voice users during the first 14 slots. These 14 slots have $8.75 \mathrm{~ms}$ of duration which leave us $11.75 \mathrm{~ms}$ remaining free of transmissions until the start of the next superframe. In a managed network a Beacon is transmitted immediately after the hop. This Beacon is used to maintain network synchronization, control the format of the superframe and manage when each node should transmit and receive data.

At the end of the first CFP in the superframe there is a space reserved for a service slot. The service slot is used by voice nodes to communicate with the Control Point. The time between the two CFPs, the contention period, is used for data transmissions using a CSMA/CA protocol similar to that specified in the 802.11 standard [4].

The MAC uses a slotted contention scheme, acknowledgement and retransmission of data messages and a fragmentation scheme to improve performance. If there is no voice connection active then the CSMA/CA period occupies the whole of the superframe, with the exception of the space required for the Beacon, maximizing data throughput. For example, 25 voice users would employ the first pair of slots for the first ten users, another pair for the next ten users and another pair for the remaining five users. That is a total of six slots $(3.75 \mathrm{~ms})$ so the data transmission can employ $16.25 \mathrm{~ms}$.

\subsection{Management}

The primary function of the Beacon is to enable all nodes to synchronize to the timing of the network. The Beacon transmitted by the access point is also used to manage the network during the contention free periods. The beacon can include a list of active voice connections (and therefore frequency hopping 
and slot assignments), retransmission slot assignments for the current superframe, connection status information and paging information. Slot assignment and synchronization information does not change on a per frame basis, so if a node misses a Beacon it uses the information contained in the most recent valid beacon.

All connection and paging status requests and information are repeated until they are acknowledged by the target node.

\section{Results}

In this section some results are presented in order to establish the benefits of Hybrid MAC proposal opposite to results obtained with $802.11 \mathrm{~g} / \mathrm{e}$. Just as an upper bound reference, we compute the effective time needed, for a VoIP transmission over $802.11 \mathrm{~g} / \mathrm{e}$. Results are shown in Table 4 . The number of admissible voice users, assuming perfect statistical multiplexing and no collisions is shown in the same table.

Table 4. Maximum capacity upper bound

\begin{tabular}{ccc} 
PHY rate Tx Time $(\mu s)$ & Voice users \\
\hline \hline 6 & 263.5 & 37.95 \\
9 & 211.5 & 47.28 \\
12 & 183.5 & 54.49 \\
18 & 159.5 & 62.69 \\
24 & 147.5 & 67.79 \\
36 & 131.5 & 76.04 \\
38 & 127.5 & 78.43 \\
54 & 123.5 & 80.97
\end{tabular}

We compare these results with the ones following the hybrid scheme (showed in Fig.4) and those obtained with the real performance of the standard WLAN. Comparison is performed assuming a variable number of VoIP users multiplexed with an FTP user transmission. Combinations of physical transmissions of $6 \mathrm{Mbps}$ and $54 \mathrm{Mbps}$ for both VoIP and FTP traffic sources have been considered. Evaluation has been made in terms of Packet Loss Probability for audio traffic and Throughput (maximum possible transmission) for the FTP source.

Figures 6(a) and 6(b) show results for an FTP physical transmission at 6Mbps multiplexed with both $6 \mathrm{Mbps}$ and $54 \mathrm{Mbps}$ for VoIP sources. We can see that in a standard WLAN environment, 50 VoIP (54Mbps) users have unacceptable levels of $15 \%$ of losses and the FTP user works at $500 \mathrm{kbps}$. In fact, maximum capacity under these conditions is 32 users (VoIP at 54Mbps) and the FTP user transmits at around $2 \mathrm{Mbps}$. However with the hybrid proposed scheme we can handle 50 VoIP users (in addition, without losses due to congestion) and 3.33Mbps for the FTP, as was expected. Note that 50 VoIP users need $5 \cdot 1.25 \mathrm{~ms}=6.25 \mathrm{~ms}$, so 


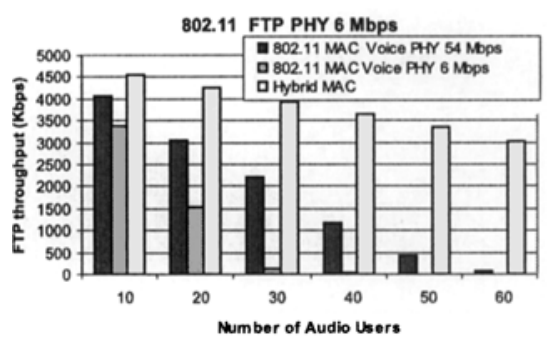

(a) FTP Throughput

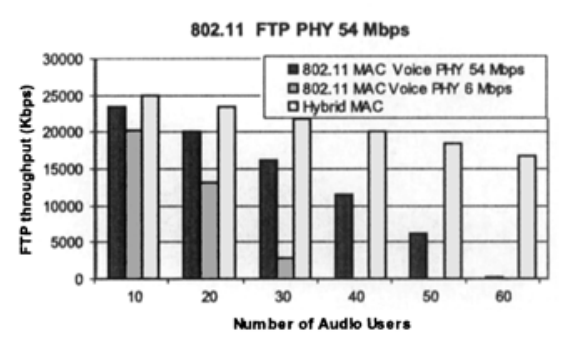

(c) FTP Throughput

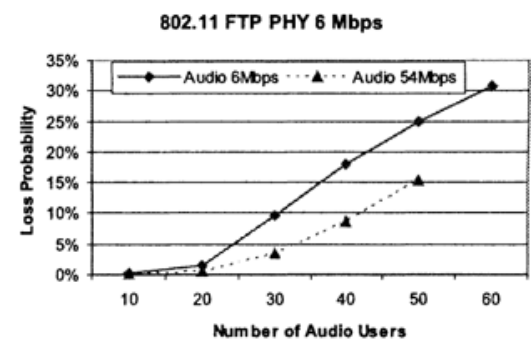

(b) Audio packet loss probability

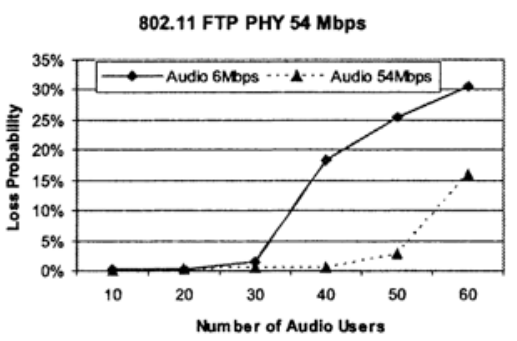

(d) Audio packet loss probability

Fig. 6. Results

13.75ms can be employed for FTP. On the other hand, effective rate when all the radio resources are assigned to the FTP transmission is $4.8 \mathrm{Mbps}$. So, if a fraction of $13.75 / 20$ of radio resources is assigned, about $3.33 \mathrm{Mbps}$ can be achieved.

In figures $6(\mathrm{c})$ and $6(\mathrm{~d})$, the same results are showed for FTP transmissions at $54 \mathrm{Mbps}$. The number of simultaneous VoIP users supposing a $5 \%$ of losses at $54 \mathrm{Mbps}$ is near 60 users and transmitting at $6 \mathrm{Mbps}$, just about 32 . The FTP throughput associated is lower than $6.2 \mathrm{Mbps}$ and $3 \mathrm{Mbps}$, respectively. However, in the hybrid case, we could even handle, without problems, 70 VoIP users and have enough remaining capacity to support a 13Mbps FTP transmission at $54 \mathrm{Mpps}$.

In all the results, the maximum number of VoIP users in the hybrid protocol outperforms even the upper bound reference considered in Table 4.

Moreover commercial $802.11 \mathrm{~g} / \mathrm{a}$ products have sensitivities in a range between $-90 \mathrm{dBm}(6 \mathrm{Mbps})$ and $-72 \mathrm{dBm}(54 \mathrm{Mbps})$ On the other hand commercial Bluetooth products require just $-85 \mathrm{dBm}$ which corresponds with a WLAN working at $9 \mathrm{Mbps}$. This fact implies that the maximum number of users supported is reduced more than a half. 


\section{Conclusions}

In this paper, a hybrid WLAN and modified Bluetooth based physical and MAC protocol layers have been proposed in order to improve system efficiency in heterogeneous traffic scenarios. Simulation results show that the new system outperforms system capacity over the WLAN-only solution when both VoIP and data transmissions are considered. Taking profit of the CSMA/CA access technique, used in WLAN, for data transmissions in addition to deterministic Bluetooth access for VoIP has a significant impact over the system capacity.

We have demonstrated along the paper that the theoretical capacity upper bound limit of a standard WLAN is overcame by the proposed solution.

The results show that not only the voice capacity of the system is improved, but also the CFP available for data transmissions, allowing a better throughput of data traffic.

\section{References}

[1] 3GPP TS 26.071, "AMR speech codec; general description"

[2] 3GPP TS 26.171, "Wideband AMR speech codec; general description"

[3] J Sjöberg et al., "RTP payload format and file storage format for the Adaptive Multi Rate (AMR) and Adaptive Multi-Rate Wideband (AMR-WB) audio codecs" 2002, IETF RFC 3267.

[4] ANSI/IEEE, 802.11-1999, "Part 11: Wireless LAN Medium Access Control (MAC) and Physical Layer (PHY) specifications", IEEE

[5] IEEE 802.11e. IEEE Standard for Information technology Telecommunications and information exchange between systems. Local and metropolitan area networks. Specific requirements Part 11: Wireless LAN Medium Access Control (MAC) and Physical Layer (PHY) specifications. Amendment 8: Medium Access Control (MAC) Quality of Service Enhancements

[6] IEEE 802.11g. IEEE Standard for Information technology. Telecommunications and information exchange between systems. Local and metropolitan area networks. Specific requirements Part 11: Wireless LAN Medium Access Control (MAC) and Physical Layer (PHY) specifications Amendment 4: Further Higher Data Rate Extension in the $2.4 \mathrm{GHz}$ Band

[7] Specification of the Bluetooth System, Core Specifications, version 2.0+EDR, v1.2, v1.1; https://www.bluetooth.org/spec/

[8] K.S.Natarajan. "A hybrid medium access control protocol for wireless LAN". 1992 IEEE International Conference on Selected Topics in Wireless Communications. pp 134-7.

[9] B. A. Sharp, E. A. Grindrod, and D. A. Camm, "Hybrid TDMA/CSMA Protocol for Self Managing Packet Radio Networks" Proc. 1995 4th IEEE ICUPC, Tokyo, Japan, Nov. 6-10, 1995, pp. 929-33.

[10] BlueCore4-External Data Sheet. http://www.csrsupport.com/BC4Ext

[11] 3GPP TS 26.201. "Technical Specification Group Services and System Aspects; Speech codec speech processing functions; Adaptive Multi-Rate - Wideband (AMRWB) speech codec; Frame structure. (Release 6)" 